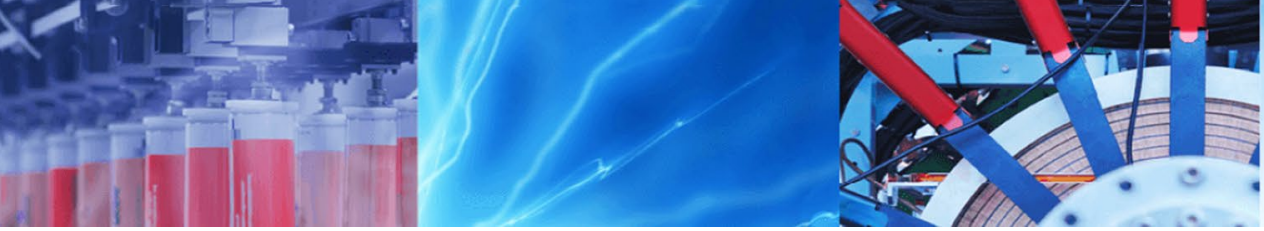

Research Article

\title{
Twin parallel channel angular extrusion as a development of ECAE in parallel channels
}

\author{
M. Abdi ${ }^{1} \cdot$ R. Ebrahimi ${ }^{1}$
}

Received: 3 November 2019 / Accepted: 25 February 2020 / Published online: 4 March 2020

(c) Springer Nature Switzerland AG 2020

\begin{abstract}
Equal Channel Angular Extrusion (ECAE) is a simple process which is suitable to deform bulk materials into extremely large strains in order to achieve ultra-fine grained (UFG) structures. However, ECAE has some limitations. In order to eliminate these shortcomings, some methods were developed including ECAE in parallel channels. In the present work, a new processing technique based on ECAE in parallel channels is introduced which is named Twin Parallel Channel Angular Extrusion. DEFORM 3D software was used to simulate and study the flow pattern during the process. In addition, the deformation of aluminum samples was conducted using this processing technique, and load-displacement curves and hardness profiles were used to confirm the validness of the simulation. The comparison of this method with the previous basic one, which is named Single Parallel Channel Angular Extrusion in this paper, was carried out. With respect to the previous method, the results show that the present one is efficient in imposing strain for more volumes of materials in addition to less energy consumption and more buckling stability of the using punch.
\end{abstract}

Keywords Finite element method - Equal channel angular extrusion · Parallel channels · Compressive stress state · Friction

\section{Introduction}

In recent years, top-down manufacturing methods based on the severe plastic deformation (SPD) have found a growing importance in order to achieve ultra-fine grained (UFG) and nano-crystalline (NC) materials [1, 2]. So many disadvantages such as porosity and impurities, which exist in bottom-up strategies like inert gas condensation [3] or melt spinning [4], are eliminated in the SPD techniques [5]. Extreme shear deformation occurs during the SPD processes, while the processed samples do not change in the cross section. Thus, it is possible to perform the process for several cycles. As a result, strain accumulation causes pile up of dislocations, leading to the formation of high-angle grain boundaries with few porosities in the final structure [6]. This ultra-grain refinement brings about exceptional mechanical properties based on the Hall-Petch theory [7]. So far, special processing techniques of severe plastic deformation have been developed and used in order to fabricate UFG metals and alloys, including equal channel angular extrusion (ECAE) [8-10], high pressure torsion (HPT) [11-13], accumulative roll bonding (ARB) [14-16], twist extrusion (TE) [17-19], simple shear extrusion [20], severe forward extrusion (SFE) [21], and constrained groove pressing (CGP) [22-24]. ECAE is one of the most well developed SPD processing techniques in which the material is deformed through a die with two channels intersecting at a certain angle, which is described completely in previous reports $[25,26]$. This technique came into use in order to produce UFG materials with unique mechanical properties in recent years [27-30], and it can be said that among all of the SPD processing techniques,

R. Ebrahimi, ebrahimy@shirazu.ac.ir|'Department of Materials Science and Engineering, School of Engineering, Shiraz University, Shiraz, Iran. 
ECAE provides the opportunity to develop processing routes and invoke different slip systems very well [31]. In order to eliminate the limitations, like removing and reinserting the samples for the following passes, of this technique, some modifications were carried out. These developments include the following: using a rotary die ECAE [32], the side-extrusion process [25], a die having multiple passes [25], and continuous ECAE [33, 34]. Using a facility containing two parallel channels was another development in which two separate shearing incidents occur through one pass of deformation, leading to a remarkable reduction of passes to achieve UFG materials [35]. In this technique, there are two main parameters changing the flow state and strain condition. These parameters are the distance between the axis of two channels, $K$, and the intersecting angle of the channels, $\Phi$. Raab [36] studied the flow state of the material through ECAE in parallel channels. Using finite element method and grid technique, different intersection angles and distances between the axis of two channels were studied. In this work, a novel processing technique is presented based on the principles of ECAE in parallel channels, [36] which is named Twin Parallel Channel Angular Extrusion (TPCAE). The strain state of the deformed material by the process is studied experimentally and numerically based on the finite element method. Moreover, the comparison between the TPCAE and the previous method, named Single Parallel Channel Angular Extrusion (SPCAE) in this work, is performed numerically, and the advantages of the new process are discussed.

\section{Materials and methods}

In this process, there is a facility containing two parts which are assembled on each other by nuts and bolts. The die facility consists of an inlet channel with a rectangular cross section and two outlet channels with square cross sections which are set apart from each other. As shown in Fig. 1a, there is a mirror symmetry with respect to the middle of the inlet channel which is placed parallel to the outlet channels. The width of the inlet channel $\left(d^{\prime}\right)$ is twice the width of each outlet channel (d), but there are all the same in other dimensions.

Two specimens with square cross section are placed side by side and are pressed simultaneously through the
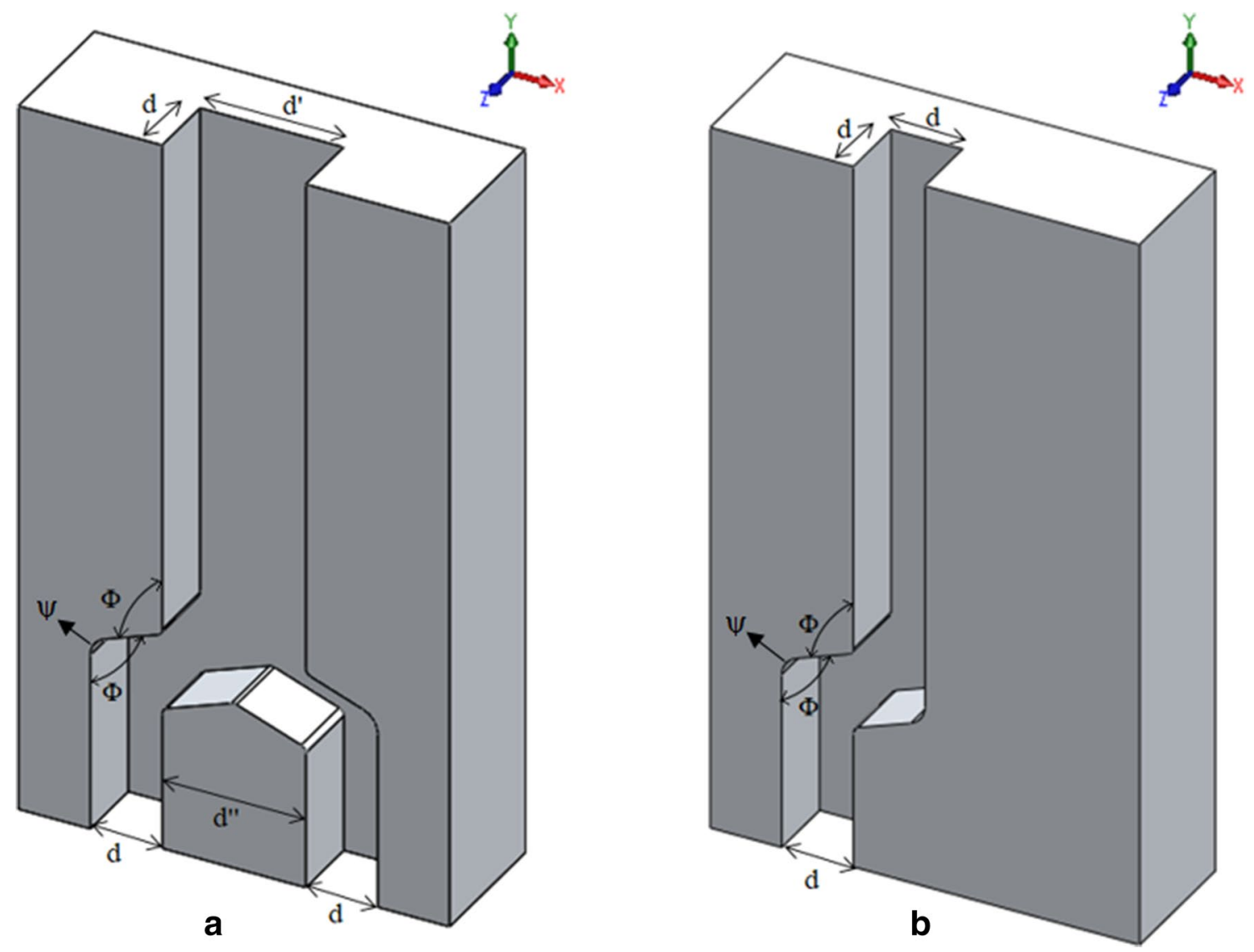

Fig. 1 Die geometry presentation of $\mathbf{a}$ TPCAE method and $\mathbf{b}$ SPCAE method 
parallel channels. The intersection angles $(\Phi)$ and the outer curved corner angles $(\psi)$ are kept $110^{\circ}$ and $18^{\circ}$ respectively, and the distance between the two outlet channels $\left(d^{\prime \prime}\right)$ equals the width of the inlet channel $\left(d^{\prime}\right)$ which means that the $\mathrm{K}$ parameter is considered equal to $\mathrm{d}$. In Fig. $1 \mathrm{~b}$, the die set for the SPCAE is shown for the sake of comparison with the twin one.

Billets of commercially pure aluminum (AA1050) with the dimensions of $10 \mathrm{~mm} \times 10 \mathrm{~mm} \times 60 \mathrm{~mm}$ cross section were prepared by machining. Then the heat treatment of annealing at $450{ }^{\circ} \mathrm{C}$ for $2 \mathrm{~h}$ and furnace cooling with a rate of $25^{\circ} \mathrm{C} / \mathrm{h}$ were conducted. After that, two billets were placed side by side in the inlet channel and deformed simultaneously with a rectangular cross sectional punch with the speed of $0.2 \mathrm{~mm} / \mathrm{s}$. The process was conducted at room temperature with a screw press, and Teflon tapes were used as a lubricant in order to reduce the friction. The displacement of the punch was kept as $45 \mathrm{~mm}$ and after that the process was stopped to show the in situ geometry of the samples. Then, one of the deformed specimens was sectioned longitudinally to investigate microhardness distribution and the flow pattern during the formation. On one of the section planes, microhardness measurements were taken on different paths. The Vickers microhardness was recorded with the applied load of $25 \mathrm{~g}$, rate of $0.2 \mathrm{~g} / \mathrm{s}$, and dwell time of $15 \mathrm{~s}$. Afterward, the other cross section plane was macroetched according to ASTM E340-15 standard to reveal the flow pattern during the process in the parallel channels. In addition, barrel compression test [37] was performed on the Teflon-wrapped compression sample with height to diameter ratio of 1.5 in order to evaluate the friction factor of the AA1050 samples in the TPCAE die.

The commercial FEM package, DEFORM 11, was considered to simulate and study the strain sate and flow behavior in the process. Simulation was conducted making a 3D model in which parts of the real die containing the inlet channel and outlet channels were designed in order to model the TPCAE, as illustrated in Fig. 1a. The friction factor at the anvil-sample interface and stress-strain relationship were obtained according to the barrel compression test [37] which simulates the lubrication condition in the experiment and flow behavior of the material based on the upper bound theory. The friction factor and stress-strain relationship were determined as 0.07 and $\sigma=106 \varepsilon^{0.347}(\mathrm{MPa})$, respectively. The dimensions of the specimens were considered according to the experiments. Therefore, it is possible to verify the simulation results with those obtained experimentally.

4-node linear tetrahedral elements were used to mesh the billets. The dies and punch were taken as rigid parts. The simulation was conducted at room temperature without considering heat transfer. Lagrangian approach was used in the finite element code with the conjugate gradient algorithm as the iterative method. According to the experiment, the die was fixed in all displacement and rotation directions and the specimens were placed in the inlet channel. The punch was kept moving in the inlet channel with the same velocity as in the experiment. In order to compare the simulation results of the TPCAE with those of the SPCAE, another die which simulates the SPCAE was designed according to the parameters considered in TPCAE, as shown in Fig. 1b. All other processing conditions such as definition of contact surfaces, deformation speed, etc. were considered the same as in the TPCAE process simulation.

\section{Results and discussion}

The in situ geometry of the deformed specimens in the TPCAE method is shown in Fig. 2. The first point that can be seen is that the deformation channels are filled completely by the deformed specimens. So, the deformed specimens can be processed in the next passes without any changes in the cross sections. In addition, there is no remarkable distortion of the initial shapes, leading to the more efficient processing of the UFG materials without so much waste materials, as presented by Raab [36]. The material flow during the process is shown in Fig. 3. As can be seen, the flow directions of material are changed at each shear

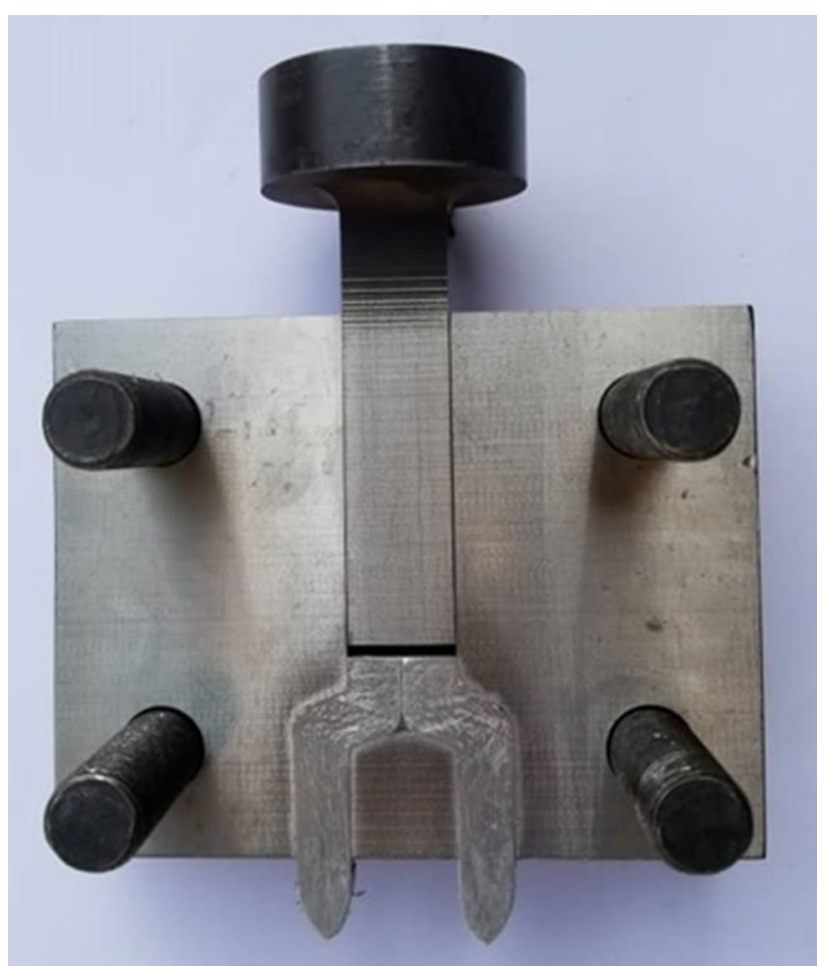

Fig. 2 The geometry of the deformed billets in the TPCAE method 


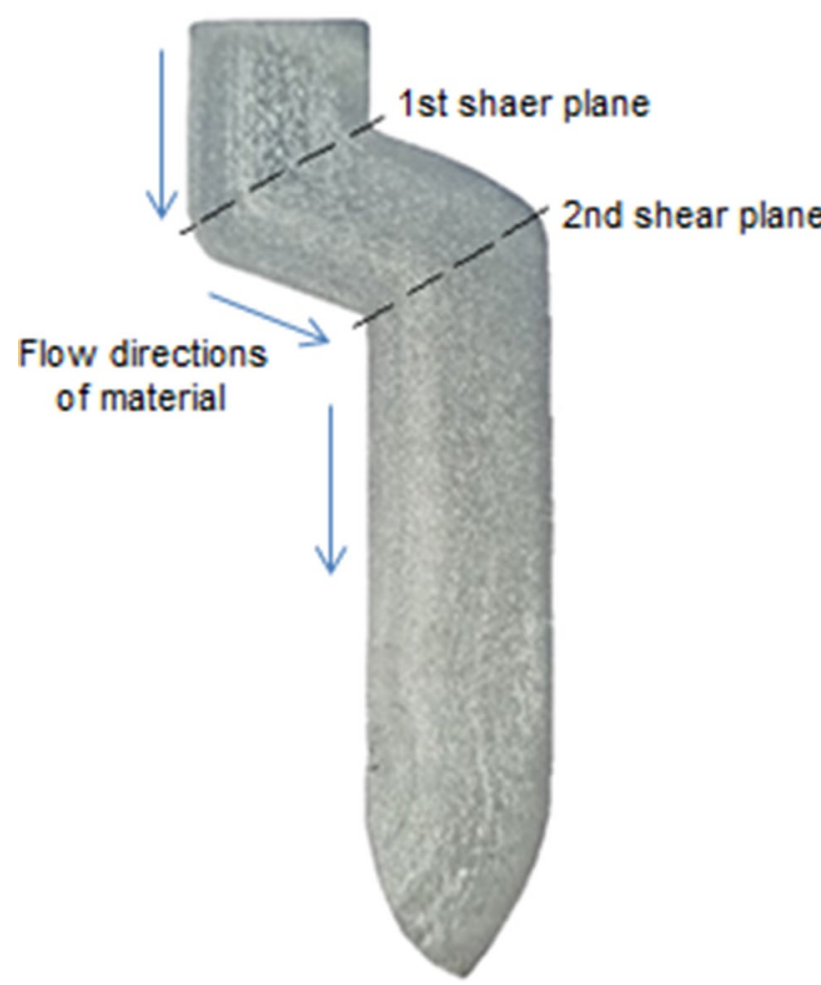

Fig. 3 Deformed specimen during the TPCAE method

planes, illustrating the shear process occurring at each shear planes. Passing two shear events at the theoretical shear planes leads to the rotation of the front surfaces of the specimens. This is in accordance with the previous reports, and makes it possible to produce equiaxed grains full of accumulated dislocations and slip lines in the UFG microstructures $[36,38]$.

The FEM simulation results of the strain fields in the cross sections of the samples during different stages of the TPCAE process are illustrated in Fig. 4. The theoretical shear planes can be observed by the sudden change of the strain magnitudes at the first and second rotations of the deformation channels. At first, the specimens pass the 1st shear plane at the first rotation of each channel and separate from each other(Fig. 4a). As the specimens are pushed ahead, they pass the 2 nd shear plane at the second rotation of each channel (Fig. 4b). By filling the outlet channels, the friction force between the contact surfaces produces a compressive stress state leading to the complete filling of the deformation zones and accumulation of more strain (Fig. 4c, d). There is a good consistency between the final shape of the processed billets (Fig. 3) and the geometry of the simulation results (Fig. 4d) illustrating the accuracy of the simulation. The important point which should be considered is that there is a homogenous strain field in the cross section of those portions which have passed two shear events with enough compressive stress state, bringing about a uniform hardness distribution and homogenous deformation in the process (Fig. 4e). The produced strain in these regions is around 1.8 on average, in agreement with the strain magnitude claimed by the previous reports [36]. Moreover, in order to compare this result with that of the theoretical strain provided in $\mathrm{ECAE}$, the following relationship is used [25]:

$\bar{\varepsilon}_{\mathrm{N}}=\frac{\mathrm{N}}{\sqrt{3}}\left(2 \cot \left(\frac{\emptyset+\psi}{2}\right)+\psi \operatorname{cosec}\left(\frac{\emptyset+\psi}{2}\right)\right)$

where $\Phi$ is the intersection angle, $\psi$ is the outer curved corner angle, and $\mathrm{N}$ is the number of passes which equals 2 here [39]. Using this relationship, the accumulated strain in the processed specimens in the outlet channels would be around 1.5. It should be considered that this formula presents the ideal deformation in ECAE and neglects some effects such as the redundant work and friction. So, the difference between the magnitudes obtained from this formula and FEM results can be attributed to the redundant work occurring during the deformation of the specimens and the friction effect in the contact surfaces of the die and specimens. As can be seen in Fig. 4, the strain is about 2.0 near the contact surfaces due to the friction effect. However, the deformation inhomogeneity in the outlet channels is found to be less than 0.5 , calculated by the standard deviation formula. The tips of the specimens experience little strain because of the lack of back pressure. However, it is obvious that methods having two shear events in each pass produce smaller tips and makes less waste materials than the traditional ECAE process, which is completely established in previous research works [36, 40]. Moreover, if two new annealed specimens are placed in the inlet channel while there are two old ones in the outlet channels, the processing of the two new specimens is conducted with more compressive stress state since old specimens hinder the forward moving of the new ones. Therefore, the front side of the processed billets would be approximately flat and the amount of waste materials decreases considerably. Figure 5 shows the Vickers microhardness values and strain distributions on the longitudinal cross section through the first pass of processing by the TPCAE method, recorded along different paths. Variation of hardness at each path illustrates that the strain is accumulated in the sample by passing two shear events in the process. At first, hardness at each path is low, which is related to the undeformed portions of the specimen. Then, hardness increases since these parts of the specimen have passed the 1st shear event. Hardness increasing continues at each path because of passing the 2 nd shear event and the existence of compressive stress state. At last, hardness at each path decreases since the front side of the billet experiences little strain due to the lack of back pressure. 
Fig. 4 Strain distribution fields obtained from FEM simulation and hardness variation achieved experimentally in the specimens processed by the TPCAE method. a-d Strain distribution at different stages of the process in the longitudinal cross sections of the billets, e strain distribution and hardness variation at the $A$ to $B$ distance in the transverse cross section of one billet
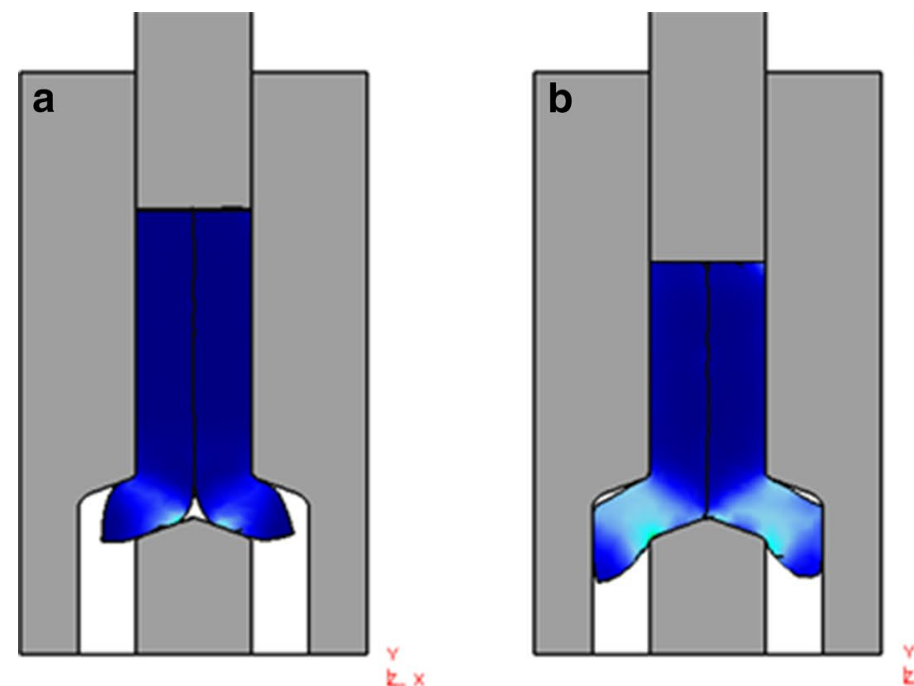

Strain-Effective

3.00

2.00

1.00

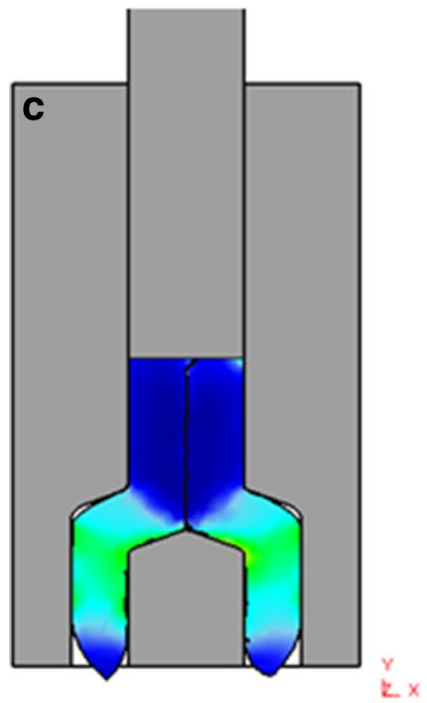

Strain-Effective

3.00

c

2.00

1.00

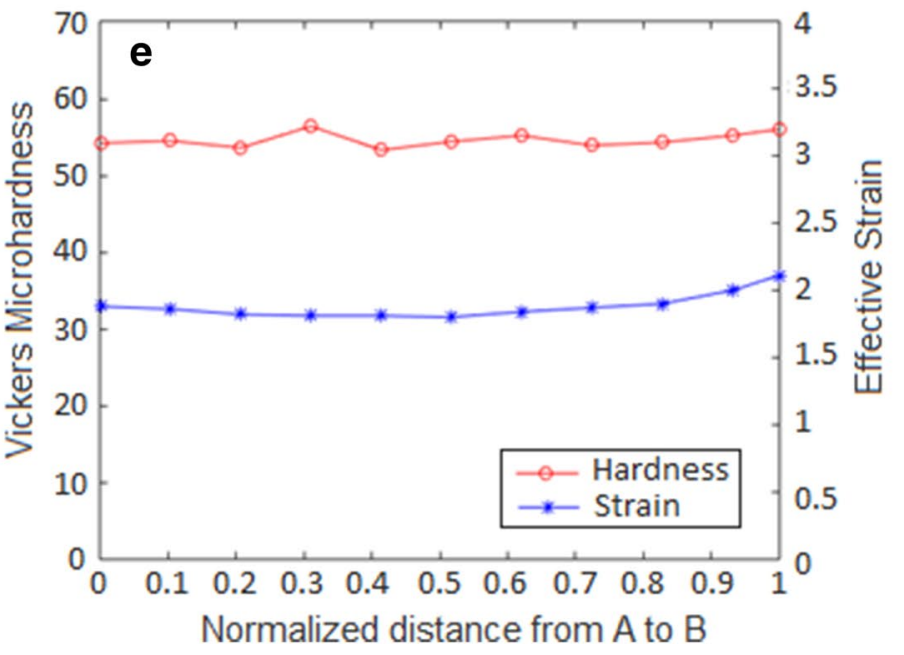

SN Applied Sciences

A SPRINGER NATURE journal 


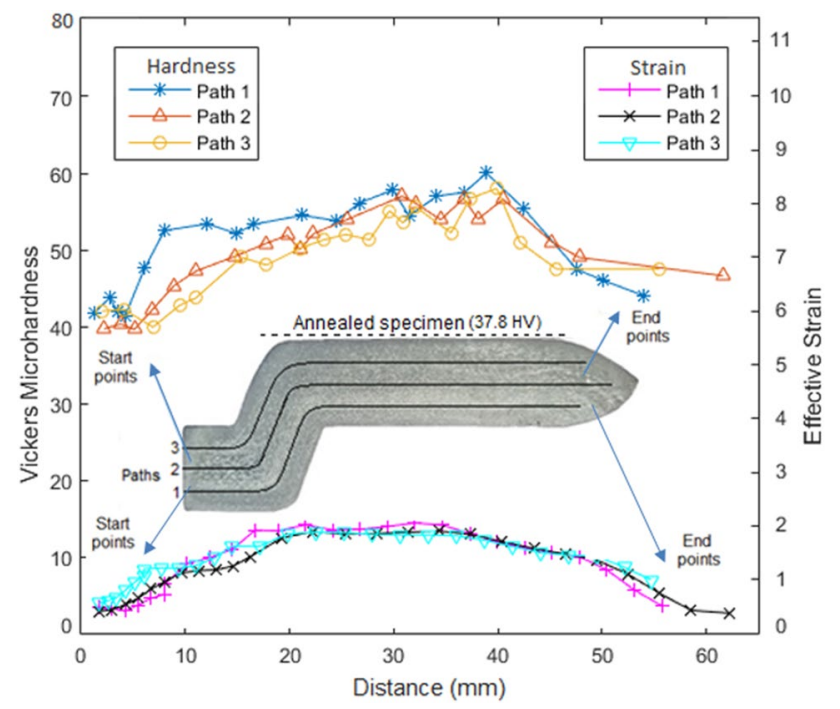

Fig. 5 Hardness and strain profiles in the longitudinal cross section of the sample processed during the TPCAE method

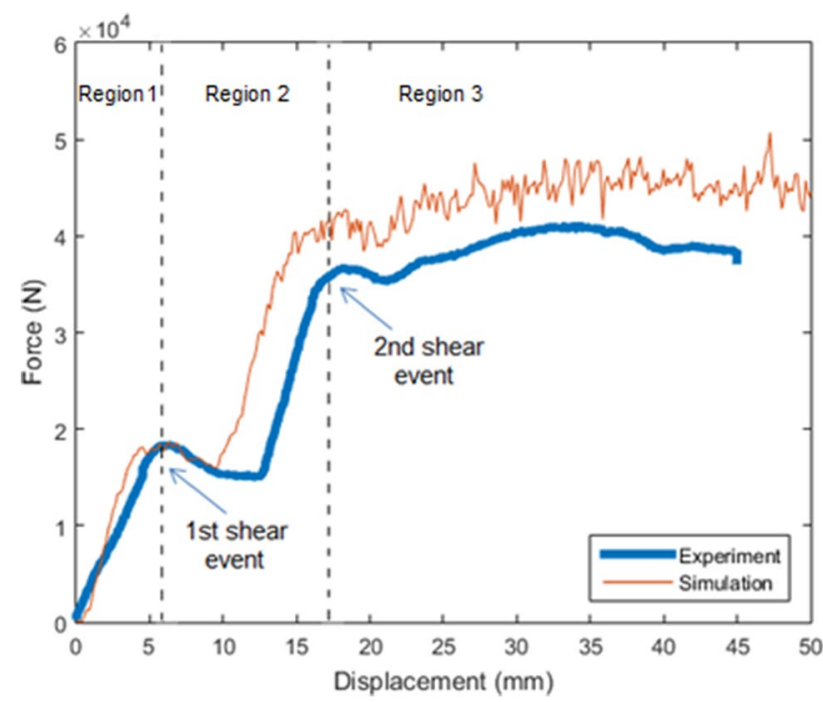

Fig. 6 Comparison of force-displacement curves obtained from experiment and FEM simulation in the TPCAE method

Increase in hardness at different paths occurs at different distances, depending on when those portions of billet materials experience the 1 st shear event. Hardness profiles, which are in agreement with the strain distributions, reveal that the material's flow pattern is highly qualitative.

Figure 6 illustrates the experimental and predicted processing load versus displacement in the TPCAE process. Acceptable consistency is seen between the trend predicted by the FEM simulation and the one obtained by the experiment. Therefore, the validity of the simulation can be totally confirmed. These curves can be divided into three regions. The first region indicates the load needed for the movement of the billets in the inlet channel. At the end of first region, there is a load peak related to the beginning of the 1st shear event for the specimens at the first rotation of the channels. The second region is related to the movement of the front side of the specimens between the 1st and 2 nd shear events. At the end of second region, there is a second load peak due to the start of the 2 nd shear event in the specimens. Actually, a load raise and subsequently a load drop can be seen at both shear events. The load raises are because of the shock applied to the specimens to start the shear events, and load drops are due to the reaching the steady state of passing billets through the shear planes. It is important to note that the shear planes in both events are similar, but the shear in the 2 nd event occurs in the opposite direction of shear in the 1st event for each sample separately. Actually, having two opposite shear events occurring subsequently in each cycle creates route $C$ automatically, similar to what is happening in ECAE with a die having multiple passes [25]. Therefore, there is no possibility to implement other routes such as route $A$ or route $\mathrm{BC}$ since the turning of samples after the 1st shear event is not feasible. Maybe, the definition of some routes can be feasible by rotating the samples after each pass, but they are not conventional route $A$ or route $B_{C}$ which are introduced in ECAE processes.

It should be said that all happenings for the two samples occur similarly since the die is symmetric and have a mirror-like geometry. Also, the lubrication conditions are kept constant throughout the channels, leading to the similar compressive stress states in the outlet channels. Therefore, the samples experience the same strain path intrinsically. The third region is related to the entering of the specimens to the outlet channels. In this region, the load increases slightly owing to the fact that the contact surfaces and friction effect increase by filling the outlet channels with deformed specimens. As the deformed specimens exit the outlet channels, the total contact surfaces and friction effect decrease, and therefore the load necessary for the accomplishment of the process decreases slightly.

Figure $7 a$ illustrates the strain field in the longitudinal cross section of the deformed specimen in SPCAE method which can be compared with the one in TPCAE method (Fig. 4d). It is illustrated that the strain distribution fields are the same in both methods and the accumulated strain in the homogenous regions is near 1.8. The difference between the two methods is related to the reduction of sliding contact surfaces. It can be said that in the TPCAE, there are fewer sliding contacts compared to the SPCAE for the constant volume of the material. This is due to the fact that in the TPCAE, the billets have no sliding movement along each other, leading to 

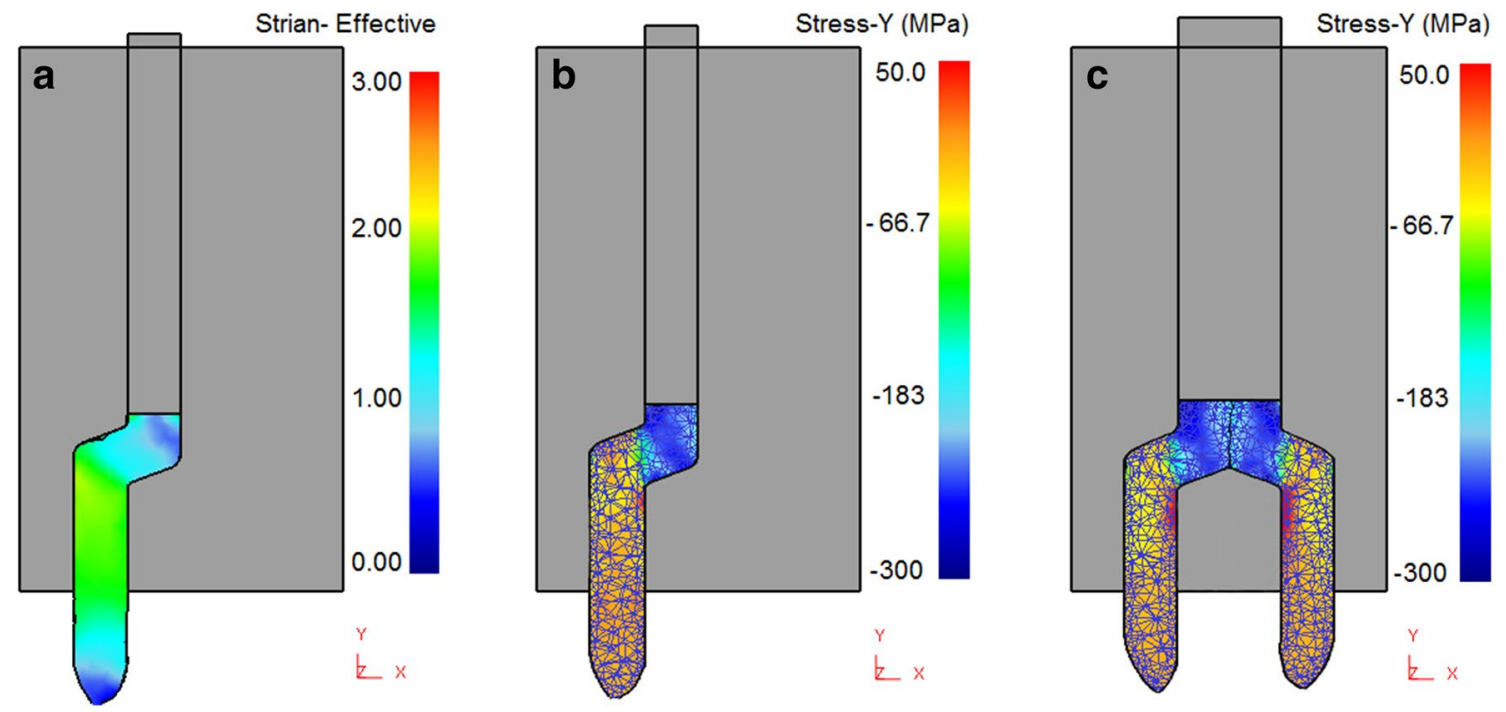

Fig. 7 Strain and stress distribution fields obtained from FEM simulation in the longitudinal cross sections of specimens. a Strain field of SPCAE, $\mathbf{b}$ stress field of SPCAE, and $\mathbf{c}$ stress field of TPCAE

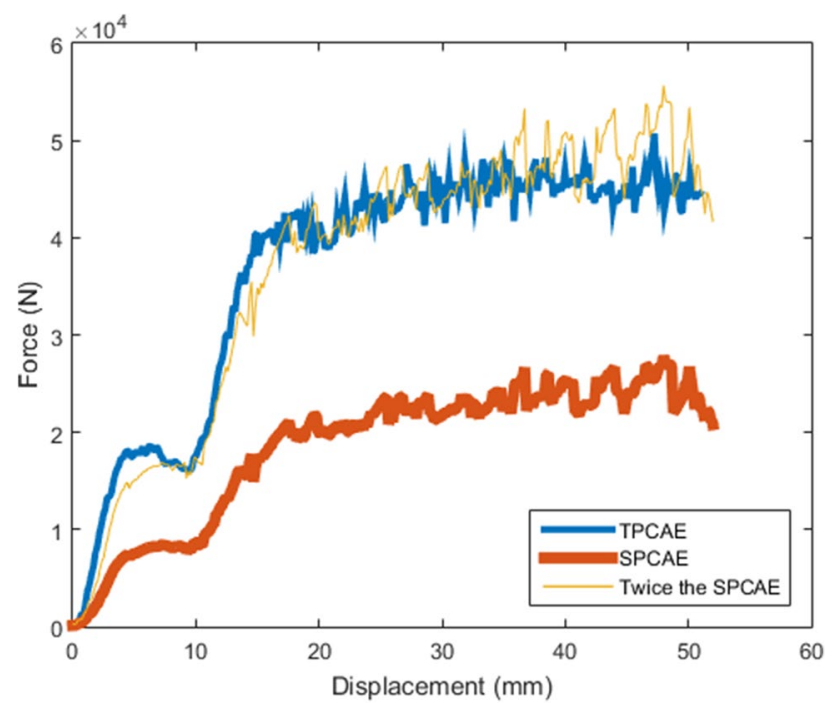

Fig. 8 Comparison of force-displacement curves obtained from FEM simulation for SPCAE and TPCAE with the friction factor of 0.07

the reduction of the contact surfaces and the reduction of energy consumption necessary for the accomplishment of the process. Figure 8 illustrates the difference in loads needed to conduct the process in the TPCAE and SPCAE with the friction factor of 0.07 in the sliding contacts. In addition, twice the load of the SPCAE is calculated and compared with the load of the TPCAE. There is approximately no significant difference between them except for the end of the process. However, this can be vital in high friction conditions. As illustrated in Fig. 9, by increasing the friction factor between the sliding

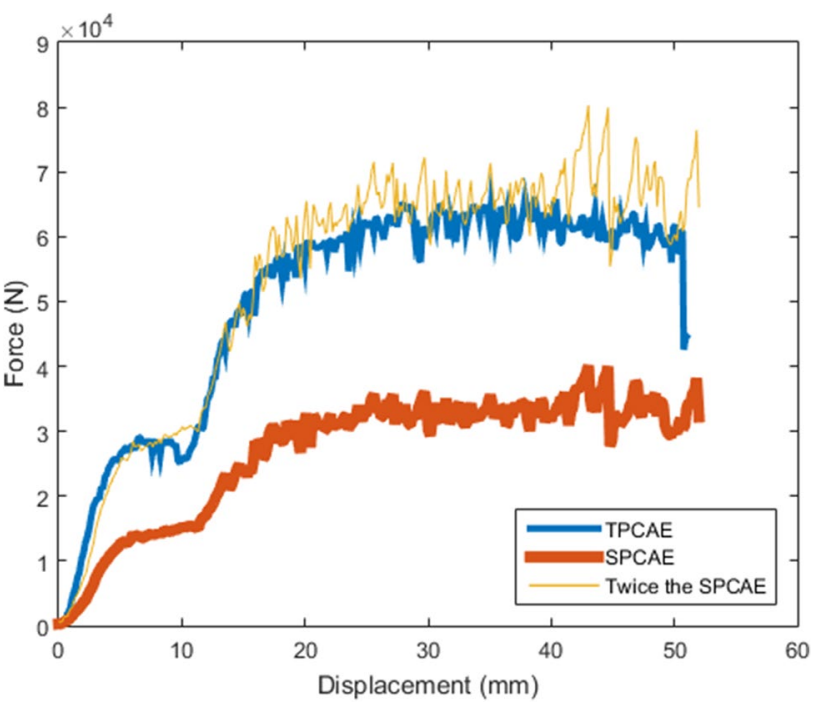

Fig. 9 Comparison of force-displacement curves obtained from FEM simulation for SPCAE and TPCAE with the friction factor of 0.15

contacts, the difference between the load of the TPCAE and twice the load of the SPCAE increases.

In high friction factors, the load of the TPCAE is approximately $7 \%$ lower than twice the load of the SPCAE due to the elimination of one contact die surface from each specimen. Instead, as mentioned before, those surfaces of each specimen are in contact with each other without any sliding movement. Thus, no energy consumption is needed in order to move those surfaces with respect to each other. In other words, more volumes of materials can be processed by the TPCAE with respect to the SPCAE, while the surface 
areas of the sliding contacts are the same and the load necessary to accomplish the TPCAE is lower than twice the load needed for the SPCAE, especially at friction factors higher than 0.1. It can be said that the TPCAE is useful at contact surfaces with high friction factors since most of the applied load is used to overcome the friction rather than the deformation itself [35].

Moreover, from die design point of view, the TPCAE can be preferred to the SPCAE considering more buckling stability of the punch according to Euler's formula. Euler's formula, which describes the critical load for the buckling of a column, can be explained by the following relationship [41]:

$p_{c r} \propto \frac{E I}{L^{2}}$

where $E$ is young modulus, $I$ is the moment of inertia of the column cross section, $L$ is the column length, and $P_{c r}$ is the critical or maximum load that can be applied to a column before it starts to buckle and collapse. Geometry and stiffness of the column have significant roles to determine critical load. Stiffness is specified by young modulus of the column and geometry is specified with length and moment of inertia produced by the column cross section.

Here, using a punch with a rectangular cross section in the TPCAE instead of a punch with a square cross section in the SPCAE leads to an increase in the moment of inertia. This increase occurs for two times along the shorter dimension and for eight times along the longer one since one of the dimensions of the rectangular cross section is twice the one in the square cross section [42]. Therefore, the critical load increases for two times and eight times along the shorter and longer dimensions of the rectangular punch cross section, respectively, while the load necessary to accomplish the process increases for less than two times. This increases the stability of the punch and its resistance to buckling.

Generally, among all different ECAE processes, the TPCAE is an attractive processing technique for several reasons, including simplicity and practicality. In addition to the advantages of the previous method [36], it provides the processing of more volumes of materials with a load lower than twice the load of the SPCAE considering constant contact surfaces. Moreover, it brings about more punch stability with respect to the SPCAE.

One has to consider that using SPCAE die with the channels two times as wide as one billet and using specimens with rectangular cross sections brings about the same advantages. However, there are some limitations with this idea. Firstly, using rectangular specimens eliminates the ability to rotate the specimens to $90^{\circ}$ and define different routes. Secondly, it is not possible to use two square specimens that are in contact with each other through all the process. Because of the asymmetric design of SPCAE, two square samples may interfere owing to the fact that they do not have sufficient rigidity such as die walls to control the material flow, or if they deform together completely, the problem of cold welding may occur. Thirdly, as illustrated in Fig. 7b, the stress distribution below the punch is asymmetric in the SPCAE method causing a bending moment that will lead to the faster instability of the punch. This asymmetric effect of stress distribution in SPCAE design will be intensified in the channels two times as wide as one billet. On the contrary, in the TPCAE method, which has a symmetric design, the stress distribution below the punch is symmetric (Fig. 7c). Hence, the distribution of the stresses are equal at both sides causing the bending moments applied to both sides of the punch compensate each other. Therefore, this symmetric stress distribution brings about more stability of the tools. From all above, it can be concluded that TPCAE design is more effective in achieving the purposes presented in this work rather than the SPCAE design with rectangular cross section channels.

In addition, by modifying this method to a new process, which may be the future work of the authors of this paper, the punch stability and its resistance to buckling can increase considerably. By the TPCAE processing of four samples with square cross sections or two samples with rectangular cross sections in parallel channels simultaneously, a new punch should be designed whose side lengths are twice in each dimension. This leads to an increase in the moment of inertia of the punch for sixteen times, while the applied load to the punch would increase for less than four times, which is due to the elimination of the contact surfaces. This leads to an increase in the punch stability for at least four times. Therefore, this new design makes this approach more potential to be presented in industrial applications.

\section{Conclusions}

In conclusion, a new SPD process, named TPCAE, is presented based on the principles of the traditional ECAE in parallel channels, and the following results are obtained:

1. This technique has the capability to impose homogenous strain of 1.8 to the material, provided that enough compressive stress state exists.

2. In addition to the advantages of the traditional ECAE in parallel channels, by employing this method, more volumes of materials per unit area of contact surfaces can be processed in each cycle with a load lower than twice the load of the traditional ECAE, especially at 
high friction factors. Consequently, less energy per unit volume is consumed in this process.

3. Punch stability and its resistance to buckling increase since the moment of inertia of the punch cross section increases for two times in one dimension and for eight times in the other dimension, while the applied load to the punch increases for less than two times. Moreover, in the symmetrical design, no bending moment is applied to the punch. This makes it more stable.

Acknowledgements The authors appreciate Shiraz University for financial support (Grant No. 97-GR-ENG 15) and research facilities used in this work.

\section{Compliance with ethical standards}

Conflict of interest On behalf of all authors, the corresponding author states that there is no conflict of interest.

\section{References}

1. Valiev RZ (2002) Materials science: nanomaterial advantage. Nature 419:887-889. https://doi.org/10.1038/419887a

2. Zehetbauer MJ, Valiev RZ (2006) Nanomaterials by severe plastic deformation. Wiley, New York

3. Chang H, Altstetter C, Averback RS (1992) Characteristics of nanophase TiAl produced by inert gas condensation. J Mater Res 7:2962-2970. https://doi.org/10.1557/JMR.1992.2962

4. Zhang LH, Duan XP, Yan X, Yu M, Ning X, Zhao Y, Long YZ (2016) Recent advances in melt electrospinning. RSC Adv 6:5340053414. https://doi.org/10.1039/C6RA09558E

5. Bagherpour E, Pardis N, Reihanian M, Ebrahimi R (2019) An overview on severe plastic deformation: research status, techniques classification, microstructure evolution, and applications. Int J Adv Manuf Technol 100:1647-1694. https://doi.org/10.1007/ s00170-018-2652-z

6. Lowe TC, Valiev RZ (2012) Investigations and applications of severe plastic deformation. Springer, Dordrecht. https://doi. org/10.1007/978-94-011-4062-1

7. Chou Y (1967) Dislocation pileups and the Hall-Petch relation. Can J Phys 45:559-566. https://doi.org/10.1139/p67-048

8. Furukawa M, Horita Z, Nemoto M, Langdon TG (2001) Processing of metals by equal-channel angular pressing. J Mater Sci 36:2835-2843. https://doi.org/10.1023/A:1017932417043

9. Tham Y, Fu M, Hng H, Pei Q, Lim K (2007) Microstructure and properties of Al-6061 alloy by equal channel angular extrusion for 16 passes. Mater Manuf Process 22:819-824. https://doi. org/10.1080/10426910701446754

10. Bagherpour E, Reihanian M, Pardis N, Ebrahimi R, Langdon TG (2018) Ten years of severe plastic deformation (SPD) in Iran, part I: equal channel angular pressing (ECAP). IJMF 5:71-113. https ://doi.org/10.22099/IJMF.2018.28756.1101

11. Liao X, Kilmametov A, Valiev R, Gao H, Li X, Mukherjee A, Bingert $J$, Zhu Y (2006) High-pressure torsion-induced grain growth in electrodeposited nanocrystalline Ni. Appl Phys Lett 88:021909. https://doi.org/10.1063/1.2159088

12. Huang J, Zhu Y, Liao X, Valiev R (2004) Amorphization of TiNi induced by high-pressure torsion. Philos Mag Lett 84:183-190. https://doi.org/10.1080/09500830310001657353
13. Liao X, Zhao Y, Zhu Y, Valiev R, Gunderov D (2004) Grain-size effect on the deformation mechanisms of nanostructured copper processed by high-pressure torsion. J Appl Phys 96:636-640. https://doi.org/10.1063/1.1757035

14. Tsuji N, Saito Y, Lee SH, Minamino Y (2003) ARB (Accumulative Roll-Bonding) and other new techniques to produce bulk ultrafine grained materials. Adv Eng Mater 5:338-344. https:// doi.org/10.1002/adem.200310077

15. Höppel HW, May J, Göken M (2004) Enhanced strength and ductility in ultrafine-grained aluminium produced by accumulative roll bonding. Adv Eng Mater 6:781-784. https://doi.org/10.1002/ adem. 200306582

16. Reihanian M, Bagherpour E, Pardis N, Ebrahimi R, Tsuji N (2018) Ten years of severe plastic deformation (SPD) in Iran, part II: accumulative roll bonding (ARB). IJMF 5:1-25. https://doi. org/10.22099/IJMF.2018.29910.1102

17. Beygelzimer $Y$, Orlov D, Korshunov A, Synkov $S$, Varyukhin $V$, Vedernikova I, Reshetov A, Synkov A, Polyakov L, Korotchenkova I (2006) Features of twist extrusion: method, structures \& material properties. Solid State Phenom 114:69-78. https://doi. org/10.4028/www.scientific.net/SSP.114.69

18. Orlov D, Beygelzimer Y, Synkov S, Varyukhin V, Tsuji N, Horita Z (2009) Microstructure evolution in pure Al processed with twist extrusion. Mater Trans 50:96-100. https://doi.org/10.2320/mater trans.MD200802

19. Varyukhin V, Beygelzimer Y, Synkov S, Orlov D (2006) Application of twist extrusion. Mater Sci Forum 503:335-340. https://doi. org/10.4028/www.scientific.net/MSF.503-504.335

20. Pardis N, Ebrahimi R (2009) Deformation behavior in Simple Shear Extrusion (SSE) as a new severe plastic deformation technique. Mater Sci Eng A 527:355-360. https://doi.org/10.1016/j. msea.2009.08.051

21. Riahi M, Ehsanian M, Asgari A, Djavanroodi F (2017) On a novel severe plastic deformation method: severe forward extrusion (SFE). Int J Adv Manuf Technol 93:1041-1050. https://doi. org/10.1007/s00170-017-0561-1

22. Zrnik J, Kovarik T, Cieslar M (2008) Microstructure and properties of aluminium processed by constrained groove pressing. Mater Sci Forum 584:535-540. https://doi.org/10.4028/www.scientific .net/MSF.584-586.535

23. Khodabakhshi F, Kazeminezhad M (2014) Differential scanning calorimetry study of constrained groove pressed low carbon steel: recovery, recrystallisation and ferrite to austenite phase transformation. Mater Sci Technol Lond 30:765-773. https://doi. org/10.1179/1743284713Y.0000000388

24. Wang Z, Liang P, Guan Y, Liu Y, Jiang L (2014) Experimental investigation of pure aluminum sheets processed by constrained groove pressing. Indian J Eng Mater S 21:121-127

25. Valiev RZ, Langdon TG (2006) Principles of equal-channel angular pressing as a processing tool for grain refinement. Prog Mater Sci 51:881-981. https://doi.org/10.1016/j.pmatsci.2006.02.003

26. Patel P, Mohanan M, Sarvaiya V, Acharya V, Basa DK, Chaudhury SK (2018) Design, fabrication of equal channel angular extrusion process and its effect on microstructure and hardness of $\mathrm{Al}$ and $\mathrm{Cu}$ alloys. T Indian I Metals 71:2605-2614. https://doi. org/10.1007/s12666-018-1396-7

27. Campbell B, Edward G (1999) Equal channel angular extrusion of polyalkenes. Plast Rubber Compos 28:467-475. https://doi. org/10.1179/146580199101540033

28. Figueiredo RB, Langdon TG (2009) Principles of grain refinement in magnesium alloys processed by equal-channel angular pressing. J Mater Sci 44:4758-4762. https://doi.org/10.1007/s1085 3-009-3725-z

29. Mostaed E, Vedani M, Hashempour M, Bestetti M (2014) Influence of ECAP process on mechanical and corrosion properties of pure Mg and ZK60 magnesium alloy for biodegradable stent 
applications. Biomatter 4:e28283. https://doi.org/10.4161/ biom.28283

30. Samigullina A, Nazarov A, Mulyukov R, Tsarenko YV, Rubanik V (2014) Effect of ultrasonic treatment on the strength and ductility of bulk nanostructured nickel processed by equal-channel angular pressing. Rev Adv Mater Sci 39:48-53

31. Abioye AA, Fayomi OSI, Popoola API, Abioye OP (2017) Equal channel angular extrusion characteristics on mechanical behavior of aluminum alloy. In: Sivasankaran S (ed) Aluminium alloysrecent trends in processing, characterization, mechanical behavior and applications, 1st edn. Intechopen, Rijeka, pp 263-277. https://doi.org/10.5772/intechopen.71019

32. Nishida Y, Arima H, Kim JC, Ando T (2000) Development of the ECAP with a rotary die and its application to AC4C aluminum alloy. J JILM 50:655-659. https://doi.org/10.2464/jilm.50.655

33. Lee JC, Chung YH, Seok HK, Suh JY, Han JH (2002) Structural evolution of a strip-cast al alloy sheet processed by continuous equal-channel angular pressing. Metall Mater Trans A 33:665673. https://doi.org/10.1007/s11661-002-0128-z

34. Zhu YT, Lowe TC, Valiev RZ, Raab GJ (2006) Continuous equal channel angular pressing. US Patent. US 7152448 B2

35. Zuyan L, Zhongjin W (1999) Finite-element analysis of the load of equal-cross-section lateral extrusion. J Mater Process Technol 94:193-196. https://doi.org/10.1016/S0924-0136(99)00096-5

36. Raab G (2005) Plastic flow at equal channel angular processing in parallel channels. Mater Sci Eng A 410:230-233. https://doi. org/10.1016/j.msea.2005.08.089
37. Ebrahimi R, Najafizadeh AA (2004) new method for evaluation of friction in bulk metal forming. J Mater Process Technol 152:136143. https://doi.org/10.1016/j.jmatprotec.2004.03.029

38. Liu Z, Liang G, Wang E, Wang Z (1998) The effect of cumulative large plastic strain on the structure and properties of a $\mathrm{Cu}-\mathrm{Zn}$ alloy. Mater Sci Eng A 242:137-140. https://doi.org/10.1016/ S0921-5093(97)00467-X

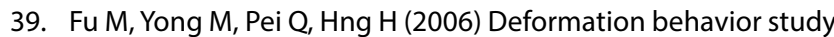
of multi-pass ECAE process for fabrication of ultrafine or nanostructured bulk materials. Mater Manuf Process 21:507-512. https://doi.org/10.1080/10426910500471557

40. Rosochowski A (2017) Severe plastic deformation technology. Whittles Publishing, Dunbeath, pp 101-104

41. Beer FP, Johnston ER, DeWolf JT (2004) Columns. In: Prasad NS, Krishnamurthy $S$ (eds) Mechanics of materials, 3rd edn. Tata McGraw-Hill, New Delhi, pp 611-612

42. Beer FP, Johnston ER, DeWolf JT (2004) Columns. In: Prasad NS, Krishnamurthy $S$ (eds) Mechanics of materials, 3rd edn. Tata McGraw-Hill, New Delhi, pp 217-218

Publisher's Note Springer Nature remains neutral with regard to jurisdictional claims in published maps and institutional affiliations. 